\title{
Finite Connectivity Attractor Neural Networks
}

\author{
B Wemmenhove ${ }^{\dagger}$ and A C C Coolen ${ }^{\ddagger}$ \\ $\dagger$ Institute for Theoretical Physics, University of Amsterdam, Valckenierstraat 65, \\ 1018 XE Amsterdam, The Netherlands \\ $\ddagger$ Department of Mathematics, King's College, University of London, The Strand, \\ London WC2R 2LS, United Kingdom
}

\begin{abstract}
We study a family of diluted attractor neural networks with a finite average number of (symmetric) connections per neuron. As in finite connectivity spin glasses, their equilibrium properties are described by order parameter functions, for which we derive an integral equation in replica symmetric (RS) approximation. A bifurcation analysis of this equation reveals the locations of the paramagnetic to recall and paramagnetic to spin-glass transition lines in the phase diagram. The line separating the retrieval phase from the spin-glass phase is calculated at zero temperature. All phase transitions are found to be continuous.

PACS numbers: 75.10.Nr, 05.20.-y, 64.60.Cn

E-mail: wemmenho@science.uva.nl, tcoolen@mth.kcl.ac.uk
\end{abstract}

\section{Introduction}

Spin models of recurrent neural networks have been studied intensively within equilibrium and non-equilibrium statistical mechanics, especially after Hopfield [1] emphasized their link with spin-glasses. In Hopfield's picture, the free energy minima of glassy systems (fixed-point attractors of the dynamics) are given an information processing interpretation: in recurrent neural networks they represent stored items of information ('patterns'), whose locations in phase space are the result of suitable modification of the neuron interactions ('learning'). Spin glass theory, especially the replica method [2], was shown to be an efficient tool with which to study the equilibrium properties of Hopfield type models (or 'attractor neural networks') with full connectivity [3. 4. It was clear from the start that full connectivity is an undesirable simplification of biological reality, made only for mathematical convenience. However, it was also clear that solving neural network models with restricted range interactions in finite dimensions $D$ was either pointless (no phase transitions for $D=1$ ) or impossible (for $D>1$ ). This dilemma prompted the study of so-called diluted models, where for each spin all but a randomly selected subset of size $c$ of interactions are removed; such can be done either while preserving interaction symmetry, or asymmetrically (in the latter case detailed balance no longer holds). Different regimes for the scaling of $c$ with the system size $N$ were found to give different physics, and to require different mathematical techniques. 
The early models of [3, 4] correspond to $c=N$. The Hopfield model with so-called extreme dilution (i.e. $\lim _{N \rightarrow \infty} c^{-1}=\lim _{N \rightarrow \infty} c / N=0$, such as $c=\log N$ ) was studied in [5] for the case of fully asymmetric dilution, and in [6] for the case of symmetric dilution. Both in fully connected and in symmetrically extremely diluted networks close to saturation one finds a conventional replica theory á la 2], with the familiar replica order parameter matrix. An alternative route away from full connectivity, which preserves both the potential for phase transitions and solvability, was followed in [7, 8] (the $1+\infty$ dimensional attractor networks). For recent reviews of the equilibrium and non-equilibrium statistical mechanics of recurrent neural networks see e.g. [9, 10].

In this paper we study the as yet unsolved class of symmetrically diluted attractor neural networks with finite connectivity, where the average number $c$ of bonds per neuron is finite, independent of the system size $N$. For bond-disordered spin systems, the finite connectivity regime (which so far has been addressed only in the context of spin glasses [11, 12, 13, 14, 16, 17, error correcting codes [18, 19] and satisfiability problems [20, 21, 22, 23] ) requires order parameter functions, which generalize the replica matrices of [2]. For finite $c$ the replica symmetry breaking theory (RSB) is still under development [16, 24, 25, 26, 17, 27]. Here we apply the finite connectivity spin glass replica techniques to a general family of attractor neural networks (which includes the Hopfield model, but also the so-called clipped Hebbian synapses, as in e.g. 28]), within the replica symmetry ansatz $(\mathrm{RS})$. We obtain phase diagrams in the $(\alpha, T)$ plane, for arbitrary finite $c$, where $\alpha=p / c$ ( $p$ giving the number of stored patterns) and $T$ is the temperature. These diagrams contain a paramagnetic phase $(\mathrm{P})$, a recall phase $(\mathrm{R})$, and a spin-glass phase (SG), all separated by second order transitions. We also analyze the RS ground state, and show how for $c \rightarrow \infty$ and arbitrary $T$ one recovers the simpler results of [6]. The most surprising outcome of our calculations is the low values of the connectivity $c$, only barely exceeding the percolation threshold, which are required for the system to operate effectively as an attractor neural network; equivalently, the robustness of such information processing systems against excessive dilution and/or physical damage.

\section{Model Definitions}

We study Ising spin neural network models, with microscopic states defined by the $N$ neuron state vector $\boldsymbol{\sigma}=\left(\sigma_{1}, \ldots, \sigma_{N}\right) \in\{-1,1\}^{N}$. Here $\sigma_{i}=1$ if neuron $i$ fires, and $\sigma_{i}=-1$ if it is at rest. Upon imposing standard Glauber-type dynamics, where the spins align stochastically to local fields of the form $h_{i}(\boldsymbol{\sigma})=\sum_{j \neq i} J_{i j} \sigma_{j}$ with symmetric interactions $\left\{J_{i j}\right\}$, these systems will evolve to thermodynamic equilibrium, described by the Hamiltonian $H(\boldsymbol{\sigma})=-\sum_{i<j}^{N} J_{i j} \sigma_{i} \sigma_{j}$ and the associated free energy

$$
F=-\beta^{-1} \log Z, \quad Z=\sum_{\boldsymbol{\sigma}} e^{-\beta H(\boldsymbol{\sigma})}
$$


The interactions are defined as a diluted and generalized version (in the spirit of [28]) of the standard Hebbian recipe, with $c_{i j} \in\{0,1\}$ and $c_{i j}=c_{j i}$ for all $(i, j)$ :

$$
J_{i j}=\frac{c_{i j}}{c} \phi\left(\sum_{\mu=1}^{p} \xi_{i}^{\mu} \xi_{j}^{\mu}\right) \quad \xi_{i}^{\mu} \in\{-1,1\} \text { for all }(i, \mu)
$$

The $p$ vectors $\boldsymbol{\xi}^{\mu}=\left(\xi_{1}^{\mu}, \ldots, \xi_{N}^{\mu}\right) \in\{-1,1\}^{N}$ represent patterns to be stored in the system. The $c_{i j} \in\{0,1\}$ define the connectivity of the network, and act as quenched disorder. They are drawn randomly and independently from

$$
P\left(c_{i j}\right)=\frac{c}{N} \delta_{c_{i j}, 1}+\left[1-\frac{c}{N}\right] \delta_{c_{i j}, 0}
$$

The average number of connections to any neuron is $c$. Averages over the realizations of the $\left\{c_{i j}\right\}$ will be denoted as $\cdots$. The function $\phi(x)$ in (2) need not be specified at this stage; for $\phi(x)=x$ one returns to the symmetrically diluted Hopfield model, for $\phi(x)=\sqrt{p} \operatorname{sgn}(x)$ one finds diluted and clipped Hebbian synapses, etc. The relevant and nontrivial scaling regime is that where $\lim _{N \rightarrow \infty} p / c=\alpha$, with $0<\alpha<\infty$.

Models of the type (2) have so far been studied in regimes where $\lim _{N \rightarrow \infty} c^{-1}=0$. Here we study the more complicated scaling regime where $c=\mathcal{O}\left(N^{0}\right)$. Our objective is to solve the model by calculating the disorder-averaged free energy per spin in the thermodynamic limit: $f=-\lim _{N \rightarrow \infty}(\beta N)^{-1} \overline{\log Z}$. The replica identity $\overline{\log Z}=$ $\lim _{n \rightarrow 0} n^{-1} \log \overline{Z^{n}}$ allows us to write this in the standard manner as

$$
f=-\lim _{N \rightarrow \infty} \lim _{n \rightarrow 0} \frac{1}{\beta N n} \log \sum_{\boldsymbol{\sigma}^{1} \ldots \boldsymbol{\sigma}^{n}} e^{\frac{\beta}{c} \sum_{i<j} c_{i j} \phi\left(\boldsymbol{\xi}_{i} \boldsymbol{\xi}_{j}\right) \sum_{\alpha} \sigma_{i}^{\alpha} \sigma_{j}^{\alpha}}
$$

Due to $c=\mathcal{O}\left(N^{0}\right)$, one can expand this expression for $N \rightarrow \infty$ :

$$
f=-\lim _{N \rightarrow \infty} \lim _{n \rightarrow 0} \frac{1}{\beta N n} \log \sum_{\boldsymbol{\sigma}^{1} \ldots \boldsymbol{\sigma}^{n}} \exp \left\{\frac{c}{2 N} \sum_{i j}\left[e^{\frac{\beta}{c} \phi\left(\boldsymbol{\xi}_{i} \cdot \boldsymbol{\xi}_{j}\right) \sum_{\alpha} \sigma_{i}^{\alpha} \sigma_{j}^{\alpha}-1}\right]+\mathcal{O}\left(N^{0}\right)\right\}
$$

In non-diluted disordered spin systems, the sum in the exponent would have been quadratic in the spin variables; there the free energy can be linearized by a gaussian transformation, leading to a single spin problem with the conventional replica order parameter matrix $\left\{q_{\alpha \beta}\right\}$ [2]. In systems with finite connectivity, in contrast, one finds

more complicated order parameters which encode higher order correlations between replicas [11. In these models it is more convenient to use an order parameter function 12, 13, 14, describing the distribution of spin variables in the various replicas, from which all the conventional order parameters can be derived.

\section{Calculation of the Free Energy}

\subsection{Replica Analysis and Sub-lattice Order Parameters}

To work out (5) we exploit the fact that for $c=\mathcal{O}\left(N^{0}\right)$, also $p=\mathcal{O}\left(N^{0}\right)$. This allows us to use the concept of sublattices [15] (of which there are $2^{p}$ ):

$$
I_{\boldsymbol{\xi}}=\left\{i \mid \boldsymbol{\xi}_{i}=\boldsymbol{\xi}\right\} \quad p_{\boldsymbol{\xi}}=\left|I_{\boldsymbol{\xi}}\right| / N
$$


Finite Connectivity Attractor Neural Networks

We define $\boldsymbol{\sigma}_{i}=\left(\sigma_{i}^{1}, \ldots, \sigma_{i}^{n}\right)$, and henceforth abbreviate averages over the sublattices as $\langle f(\boldsymbol{\xi})\rangle_{\boldsymbol{\xi}}=\sum_{\boldsymbol{\xi}} p_{\boldsymbol{\xi}} f(\boldsymbol{\xi})$. The trace in (5) can now be written as

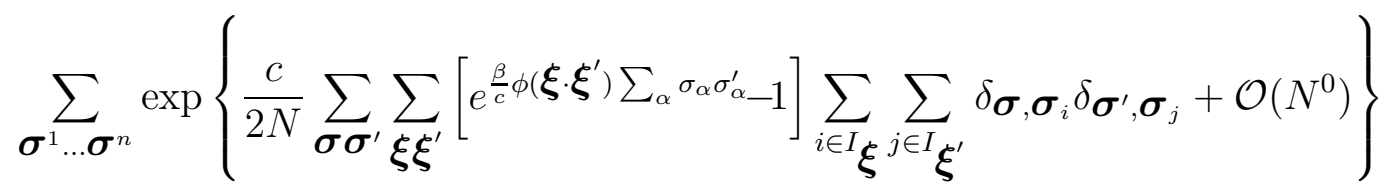

We next introduce a spin distribution $P_{\boldsymbol{\xi}}(\boldsymbol{\sigma})$ within each sublattice, with $\boldsymbol{\sigma}=$ $\left\{\sigma^{1}, \ldots, \sigma^{n}\right\}$, to be isolated upon inserting suitable $\delta$-distributions into (5):

$$
1=\int \prod_{\boldsymbol{\xi} \boldsymbol{\sigma}}\left\{d P_{\boldsymbol{\xi}}(\boldsymbol{\sigma}) \delta\left[P_{\boldsymbol{\xi}}(\boldsymbol{\sigma})-\frac{1}{\left|I_{\boldsymbol{\xi}}\right|} \sum_{i \in I_{\boldsymbol{\xi}}} \delta \boldsymbol{\sigma}_{,} \boldsymbol{\sigma}_{i}\right]\right\}
$$

From these distributions, one derives the more familiar types of observables such as replicated sublattice magnetizations $m_{\boldsymbol{\xi}}^{\alpha}$ and replicated pattern overlaps $m^{\mu \alpha}$ :

$$
m_{\boldsymbol{\xi}}^{\alpha}=\sum_{\boldsymbol{\sigma}} P_{\boldsymbol{\xi}}(\boldsymbol{\sigma}) \sigma^{\alpha} \quad m^{\mu \alpha}=\left\langle\xi^{\mu} m_{\boldsymbol{\xi}}^{\alpha}\right\rangle_{\boldsymbol{\xi}}
$$

The introduction of integral representations for the delta functions in (8) generates conjugate variables $\hat{P}_{\boldsymbol{\xi}}(\boldsymbol{\sigma})$. The trace in (17) is now performed trivially, resulting in an integral to be evaluated by steepest descent:

$$
\begin{aligned}
& f=-\lim _{N \rightarrow \infty} \lim _{n \rightarrow 0} \frac{1}{\beta N n} \log \int\left[\prod_{\boldsymbol{\xi} \boldsymbol{\sigma}} d P_{\boldsymbol{\xi}}(\boldsymbol{\sigma}) d \hat{P}_{\boldsymbol{\xi}}(\boldsymbol{\sigma})\right] \exp \left\{i N \sum_{\boldsymbol{\sigma}}\left\langle\hat{P}_{\boldsymbol{\xi}}(\boldsymbol{\sigma}) P_{\boldsymbol{\xi}}(\boldsymbol{\sigma})\right\rangle_{\boldsymbol{\xi}}\right\} \\
& \times \exp \left\{\frac{c N}{2}\left\langle\left\langle\sum_{\boldsymbol{\sigma} \boldsymbol{\sigma}^{\prime}} P_{\boldsymbol{\xi}}(\boldsymbol{\sigma}) P_{\boldsymbol{\xi}^{\prime}}\left(\boldsymbol{\sigma}^{\prime}\right)\left[e^{\frac{\beta}{c} \phi\left(\boldsymbol{\xi} \cdot \boldsymbol{\xi}^{\prime}\right)\left(\boldsymbol{\sigma} \cdot \boldsymbol{\sigma}^{\prime}\right)-1}\right]\right\rangle\right\rangle \boldsymbol{\xi}^{\prime}+\mathcal{O}\left(N^{0}\right)\right\} \\
& \times \exp \left\{N\left\langle\log \left[\sum_{\boldsymbol{\sigma}} e^{-i \hat{P}(\boldsymbol{\sigma})}\right]\right\rangle \boldsymbol{\xi}\right\} \\
& =-\lim _{n \rightarrow 0} \frac{1}{\beta n} \operatorname{extr}_{\left\{P_{\boldsymbol{\xi}}(\boldsymbol{\sigma}), \hat{P}_{\boldsymbol{\xi}}(\boldsymbol{\sigma})\right\}}\left\{i \sum_{\boldsymbol{\sigma}}\left\langle\hat{P}_{\boldsymbol{\xi}}(\boldsymbol{\sigma}) P_{\boldsymbol{\xi}}(\boldsymbol{\sigma})\right\rangle_{\boldsymbol{\xi}}+\left\langle\log \left[\sum_{\boldsymbol{\sigma}} e^{-i \hat{P}^{(\boldsymbol{\sigma})}}\right]\right\rangle \boldsymbol{\xi}\right. \\
& \left.+\frac{1}{2} c\left\langle\left\langle\sum_{\boldsymbol{\sigma} \boldsymbol{\sigma}^{\prime}} P_{\boldsymbol{\xi}}(\boldsymbol{\sigma}) P_{\boldsymbol{\xi}^{\prime}}\left(\boldsymbol{\sigma}^{\prime}\right)\left[e^{\frac{\beta}{c} \phi\left(\boldsymbol{\xi} \cdot \boldsymbol{\xi}^{\prime}\right)\left(\boldsymbol{\sigma} \cdot \boldsymbol{\sigma}^{\prime}\right)}-1\right]\right\rangle\right\rangle \boldsymbol{\xi} \boldsymbol{\xi}^{\prime}\right\}
\end{aligned}
$$

Variation with respect to $\left\{P_{\boldsymbol{\xi}}(\boldsymbol{\sigma})\right\}$ gives an equation with which to eliminate the

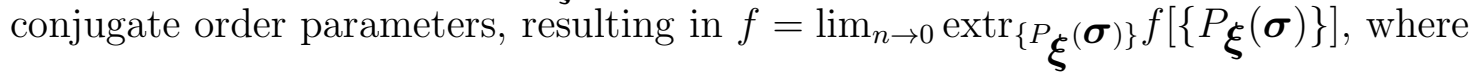

$$
\begin{aligned}
& f[\ldots]=\frac{c}{2 \beta n}\left\langle\left\langle\sum_{\boldsymbol{\sigma} \boldsymbol{\sigma}^{\prime}} P_{\boldsymbol{\xi}}(\boldsymbol{\sigma}) P_{\boldsymbol{\xi}^{\prime}}\left(\boldsymbol{\sigma}^{\prime}\right)\left[e^{\frac{\beta}{c} \phi\left(\boldsymbol{\xi} \cdot \boldsymbol{\xi}^{\prime}\right)\left(\boldsymbol{\sigma} \cdot \boldsymbol{\sigma}^{\prime}\right)}-1\right]\right\rangle\right\rangle_{\boldsymbol{\xi} \boldsymbol{\xi}^{\prime}} \\
& -\frac{1}{\beta n}\left\langle\log \left[\sum_{\boldsymbol{\sigma}} \exp \left\{c\left\langle\sum_{\boldsymbol{\sigma}^{\prime}} P_{\boldsymbol{\xi}^{\prime}}\left(\boldsymbol{\sigma}^{\prime}\right)\left[e^{\frac{\beta}{c} \phi\left(\boldsymbol{\xi} \cdot \boldsymbol{\xi}^{\prime}\right)\left(\boldsymbol{\sigma} \cdot \boldsymbol{\sigma}^{\prime}\right)}-1\right]\right\rangle \boldsymbol{\xi}^{\prime}\right\}\right]\right\rangle
\end{aligned}
$$

Further variation, again with respect to $P_{\boldsymbol{\xi}}(\boldsymbol{\sigma})$, then leads to the saddle point equation

$$
P_{\boldsymbol{\xi}}(\boldsymbol{\sigma})=\frac{\exp \left\{c\left\langle\sum \boldsymbol{\sigma}^{\prime} P_{\boldsymbol{\xi}^{\prime}}\left(\boldsymbol{\sigma}^{\prime}\right)\left[e^{\frac{\beta}{c} \phi\left(\boldsymbol{\xi} \cdot \boldsymbol{\xi}^{\prime}\right)\left(\boldsymbol{\sigma} \cdot \boldsymbol{\sigma}^{\prime}\right)}-1\right]\right\rangle \boldsymbol{\xi}^{\prime}\right\}}{\sum \boldsymbol{\sigma}^{\prime} \exp \left\{c\left\langle\sum \boldsymbol{\sigma}^{\prime \prime} P_{\boldsymbol{\xi}^{\prime \prime}}\left(\boldsymbol{\sigma}^{\prime \prime}\right)\left[e^{\frac{\beta}{c} \phi\left(\boldsymbol{\xi}^{\prime \prime} \cdot \boldsymbol{\xi}\right)\left(\boldsymbol{\sigma}^{\prime \prime} \cdot \boldsymbol{\sigma}^{\prime}\right)}-1\right]\right\rangle \boldsymbol{\xi}^{\prime \prime}\right\}}
$$


When deriving (12) it first appears that one must also allow for adding to $\left\{P_{\boldsymbol{\xi}}(\boldsymbol{\sigma})\right\}$ any vector in the kernel of the $2^{p+n} \times 2^{p+n}$ matrix $U\left(\boldsymbol{\xi}, \boldsymbol{\sigma} ; \boldsymbol{\xi}^{\prime}, \boldsymbol{\sigma}^{\prime}\right)=\exp \left[\frac{\beta}{c} \phi\left(\boldsymbol{\xi} \cdot \boldsymbol{\xi}^{\prime}\right)\left(\boldsymbol{\sigma} \cdot \boldsymbol{\sigma}^{\prime}\right)\right]-1$. However, adding such elements is seen to generate new expressions for $\left\{P_{\boldsymbol{\xi}}(\boldsymbol{\sigma})\right\}$ which again solve (12); hence (12) is always satisfied at the saddle-point.

An alternative route for calculating the free energy will turn out to be more convenient for calculating zero temperature properties, such as ground state energy and entropy. Here we first vary (10) with respect to $\hat{P}_{\boldsymbol{\xi}}(\boldsymbol{\sigma})$, resulting in $f=$ $\left.\lim _{n \rightarrow 0} \operatorname{extr}_{\{P}(\boldsymbol{\sigma})\right\} f\left[\left\{P_{\boldsymbol{\xi}}(\boldsymbol{\sigma})\right\}\right]$ subject to the constraints $\sum \boldsymbol{\sigma} P_{\boldsymbol{\xi}}(\boldsymbol{\sigma})=1$ for all $\boldsymbol{\xi}$, with

$$
\begin{aligned}
f[\ldots]= & -\frac{c}{2 \beta n}\left\langle\left\langle\sum_{\boldsymbol{\sigma} \boldsymbol{\sigma}^{\prime}} P_{\boldsymbol{\xi}}(\boldsymbol{\sigma}) P_{\boldsymbol{\xi}^{\prime}}\left(\boldsymbol{\sigma}^{\prime}\right)\left[e^{\frac{\beta}{c} \phi\left(\boldsymbol{\xi} \cdot \boldsymbol{\xi}^{\prime}\right)\left(\boldsymbol{\sigma} \cdot \boldsymbol{\sigma}^{\prime}\right)}-1\right]\right\rangle\right\rangle_{\boldsymbol{\xi} \boldsymbol{\xi}^{\prime}} \\
& +\frac{1}{\beta n}\left\langle\sum_{\boldsymbol{\sigma}} P_{\boldsymbol{\xi}}(\boldsymbol{\sigma}) \log P_{\boldsymbol{\xi}}(\boldsymbol{\sigma})\right\rangle_{\boldsymbol{\xi}}
\end{aligned}
$$

The conjugate order parameter functions $\hat{P}_{\boldsymbol{\xi}}(\boldsymbol{\sigma})$ are now found to act as Lagrange multipliers, imposing the normalization constraints on the distributions $P_{\boldsymbol{\xi}}(\boldsymbol{\sigma})$.

\subsection{RS Order Parameter Equations and Free Energy}

In order to take the $n \rightarrow 0$ limit we make the replica symmetric (RS) ansatz for the order parameters. One anticipates RS to be broken in the low temperature phases [1, 11, 6], but to hold up to the first phase transition away from the paramagnetic phase, as in the Viana-Bray model [11. In the present context, $\mathrm{RS}$ is equivalent to assuming that the $P_{\boldsymbol{\xi}}(\boldsymbol{\sigma})$ are invariant under permutations of the components of $\boldsymbol{\sigma}$, i.e. only depend on the sum $\sum_{\alpha} \sigma^{\alpha}$. Upon defining effective fields [12], viz. $h_{i} \equiv \frac{1}{\beta} \tanh ^{-1}\left\langle\sigma_{i}^{\alpha}\right\rangle_{\beta}$, our RS order parameter functions can thus be written [17] as

$$
P_{\boldsymbol{\xi}}(\boldsymbol{\sigma})=\int d h W_{\boldsymbol{\xi}}(h) \frac{e^{\beta h \sum_{\alpha} \sigma^{\alpha}}}{[2 \cosh (\beta h)]^{n}}
$$

Here $W_{\boldsymbol{\xi}}(h) \geq 0$ and $\int d h W_{\boldsymbol{\xi}}(h)=1$. One can write sub-lattice magnetizations and higher order observables in terms of the $W_{\boldsymbol{\xi}}(h)$ :

$$
\sum_{\boldsymbol{\sigma}} P_{\boldsymbol{\xi}}(\boldsymbol{\sigma}) \sigma^{\alpha} \sigma^{\beta} \ldots \sigma^{r}=\int d h W_{\boldsymbol{\xi}}(h) \tanh ^{r}(\beta h) \quad(\alpha<\beta<\ldots<r)
$$

The pattern overlaps $m^{\mu \alpha}$ are now independent of $\alpha, m^{\mu \alpha}=m_{\mu}$, where

$$
m_{\mu}=\left\langle\xi^{\mu} \int d h W_{\boldsymbol{\xi}}(h) \tanh (\beta h)\right\rangle_{\boldsymbol{\xi}}
$$

We substitute (14) into (12), and isolate the occurrences of $\sum_{\alpha} \sigma^{\alpha}$ by inserting

$$
1=\sum_{m=-\infty}^{\infty} \int_{0}^{2 \pi} \frac{d \hat{m}}{2 \pi} e^{i \hat{m}\left(m-\sum_{\alpha} \sigma^{\alpha}\right)}
$$

After some mostly straightforward manipulations $\ddagger$ one can then take the $n \rightarrow 0$ limit and find (12) converting into:

$$
\int d h W_{\boldsymbol{\xi}}(h) e^{\beta h m}=\exp \left\{c\left\langle\int d h^{\prime} W_{\boldsymbol{\xi}^{\prime}}\left(h^{\prime}\right)\left[e^{m \tanh ^{-1}\left[\tanh \left(\beta h^{\prime}\right) \tanh \left(\frac{\beta}{c} \phi\left(\boldsymbol{\xi} \cdot \boldsymbol{\xi}^{\prime}\right)\right)\right]}-1\right]\right\rangle \boldsymbol{\xi}^{\prime}\right\}
$$

$\ddagger$ The only technical subtlety is the need to take $n \rightarrow 0$ for even $n$, to avoid in the denominator of (12) tricky terms like $\log \cos (\hat{m})$ with $\hat{m}<0$. The same issue arises when calculating the RS free energy. 
This is to hold for any real $m$. Provided the various integrals exist, we may now also put $m \rightarrow i m / \beta$ and carry out an inverse Fourier transform (following [17), leading to

$$
\begin{aligned}
& W_{\boldsymbol{\xi}}(h)=\int \frac{d m}{2 \pi} e^{-i m h} \\
& \quad \times \exp \left\{c\left\langle\int d h^{\prime} W_{\boldsymbol{\xi}^{\prime}}\left(h^{\prime}\right)\left[e^{\frac{i m}{\beta} \tanh ^{-1}\left[\tanh \left(\beta h^{\prime}\right) \tanh \left(\frac{\beta}{c} \phi\left(\boldsymbol{\xi} \cdot \boldsymbol{\xi}^{\prime}\right)\right)\right]}-1\right]\right\rangle \boldsymbol{\xi}^{\prime}\right\}
\end{aligned}
$$

These are the final equations from which to solve the RS order parameters, i.e. the $2^{p}$ effective field distributions $W_{\boldsymbol{\xi}}(h)$.

Upon inserting the RS ansatz (14) into expression (11) for the free energy, one can again take the limit $n \rightarrow 0$ (provided $n$ is even). However, the calculation of $f$ is found to be easier using the alternative expression (13) (see Appendix A for details). The result of the calculation is

$$
\begin{aligned}
f= & \frac{c}{2 \beta}\left\langle\left\langle\int d h d h^{\prime} W_{\boldsymbol{\xi}}(h) W_{\boldsymbol{\xi}^{\prime}}\left(h^{\prime}\right) \log \left[1+\tanh (\beta h) \tanh \left(\beta h^{\prime}\right) \tanh \left(\frac{\beta}{c} \phi\left(\boldsymbol{\xi} \cdot \boldsymbol{\xi}^{\prime}\right)\right)\right]\right\rangle\right\rangle_{\boldsymbol{\xi} \boldsymbol{\xi}^{\prime}} \\
& -\frac{c}{2 \beta}\left\langle\left\langle\log \cosh \left[\frac{\beta}{c} \phi\left(\boldsymbol{\xi} \cdot \boldsymbol{\xi}^{\prime}\right)\right]\right\rangle\right\rangle_{\boldsymbol{\xi} \boldsymbol{\xi}^{\prime}}-\frac{1}{\beta}\left\langle\int d h W_{\boldsymbol{\xi}}(h) \log [2 \cosh (\beta h)]\right\rangle_{\boldsymbol{\xi}} \\
& -\frac{c}{2 \beta}\left\langle\left\langle\int d h W_{\boldsymbol{\xi}}(h) \log \left[1-\tanh ^{2}(\beta h) \tanh ^{2}\left(\frac{\beta}{c} \phi\left(\boldsymbol{\xi} \cdot \boldsymbol{\xi}^{\prime}\right)\right)\right]\right\rangle\right\rangle_{\boldsymbol{\xi} \boldsymbol{\xi}^{\prime}}
\end{aligned}
$$

In deriving (20), which is reassuringly similar but not identical to the corresponding expression derived for the RS free energy of spin glasses with random interactions [13], we have used $W_{\boldsymbol{\xi}}(h)$ solving (19) and being normalized (for every $\boldsymbol{\xi}$ ).

As a simple test of our RS results, one may inspect the limit $c \rightarrow \infty$ for $\phi(x)=x$. Here the interactions are of the Hopfield type, and, since $p$ is finite, one should recover the equations describing the Hopfield model away from saturation. Expansion of the saddle-point equation (19) in powers of $1 / c$, keeping only the $\mathcal{O}\left(\left(\frac{1}{c}\right)^{0}\right)$ terms, results in

$$
W_{\boldsymbol{\xi}}(h)=\delta\left(h-\sum_{\mu} \xi^{\mu} m^{\mu}\right)
$$

Upon using this expression to calculate the overlaps $m^{\mu}=\left\langle\xi^{\mu} \int d h W_{\boldsymbol{\xi}}(h) \tanh (\beta h)\right\rangle_{\boldsymbol{\xi}}$ one indeed recovers the saddle-point equations of the Hopfield model away from saturation. Similarly, (20) reduces to the correct corresponding free energy.

\section{Analysis of Phase Transitions}

\subsection{Expansion of the saddle-point equations}

The paramagnetic state $W_{\boldsymbol{\xi}}(h)=\delta(h) \forall \boldsymbol{\xi}$ always solves (19). As the temperature is lowered from $T=\infty$, we expect other solutions to bifurcate away from the paramagnetic one. In finite connectivity spin glasses, phase transitions were found upon expanding the RS free energy in the order parameters (15) near the paramagnetic phase [1]. A similar strategy can be applied here, following [12. We assume that, close to the transition, $\int d h W_{\boldsymbol{\xi}}(h) h^{\ell}=\mathcal{O}\left(\epsilon^{\ell}\right)$ with $0<\epsilon \ll 1$, and we expand both sides of (18) in powers of $\epsilon$. 
This can be done self-consistently, for all al orders in $\epsilon$. Identification of the lowest two orders $\epsilon$ and $\epsilon^{2}$ is then found to give, respectively

$$
\begin{aligned}
& \int d h W_{\boldsymbol{\xi}}(h) h=c\left\langle\int d h W_{\boldsymbol{\xi}^{\prime}}(h) h \tanh \left[\frac{\beta}{c} \phi\left(\boldsymbol{\xi} \cdot \boldsymbol{\xi}^{\prime}\right)\right]\right\rangle_{\boldsymbol{\xi}^{\prime}} \\
& \int d h W_{\boldsymbol{\xi}}(h) h^{2}-\left[\int d h W_{\boldsymbol{\xi}}(h) h\right]^{2}=c\left\langle\int d h W_{\boldsymbol{\xi}^{\prime}}(h) h^{2} \tanh ^{2}\left[\frac{\beta}{c} \phi\left(\boldsymbol{\xi} \cdot \boldsymbol{\xi}^{\prime}\right)\right]\right\rangle_{\boldsymbol{\xi}^{\prime}}
\end{aligned}
$$

There are hence two types of transitions away from the paramagnetic $(\mathrm{P})$ state. The first corresponds to the lowest order being $\epsilon$; such transitions are marked by the appearance of nonzero solutions of (22). The second type have $\epsilon^{2}$ as lowest non-zero order; these transitions are marked by non-trivial solutions of (23) with $\int d h W_{\boldsymbol{\xi}}(h) h=0$. We conclude that these transitions are marked by the highest temperature for which the two $2^{p} \times 2^{p}$ matrices $M_{\boldsymbol{\xi} \boldsymbol{\xi}^{\prime}}$ and $Q_{\boldsymbol{\xi} \boldsymbol{\xi}^{\prime}}$, respectively, have an eigenvalue equal to 1 :

$$
M_{\boldsymbol{\xi} \boldsymbol{\xi}^{\prime}}=c p_{\boldsymbol{\xi}^{\prime}} \tanh \left[\frac{\beta}{c} \phi\left(\boldsymbol{\xi} \cdot \boldsymbol{\xi}^{\prime}\right)\right] \quad Q_{\boldsymbol{\xi} \boldsymbol{\xi}^{\prime}}=c p_{\boldsymbol{\xi}^{\prime}} \tanh ^{2}\left[\frac{\beta}{c} \phi\left(\boldsymbol{\xi} \cdot \boldsymbol{\xi}^{\prime}\right)\right]
$$

It follows from (16) that bifurcation of $M$ eigenvectors corresponds to a transition towards a retrieval state $(\mathrm{R})$, with nonzero pattern overlaps, whereas bifurcation of $Q$ eigenvectors corresponds to a transition towards a spin-glass state (SG).

\subsection{Transition Temperatures for Random Patterns}

For independently drawn random patterns, where $p_{\boldsymbol{\xi}}=2^{-p}$ for all $\boldsymbol{\xi}$, both matrices in (24) are symmetric and can be diagonalized by exploiting symmetries. The desired eigenvectors have in fact already been calculated in [28] (in a different context). They are found to be universal for all $2^{p} \times 2^{p}$ symmetric matrices whose entries depend only on $\boldsymbol{\xi} \cdot \boldsymbol{\xi}^{\prime}$, i.e. $U_{\boldsymbol{\xi} \boldsymbol{\xi}^{\prime}}=U\left(\boldsymbol{\xi} \cdot \boldsymbol{\xi}^{\prime}\right)$. With each of the $2^{p}$ index subsets $S \subseteq\{1,2, \ldots, p\}$ one can associate an eigenvector $v_{S}$, defined as $v_{S}(\boldsymbol{\xi})=\prod_{\mu \in S} \xi^{\mu}$. The eigenvector corresponding to the empty set is defined as $v_{\emptyset}(\boldsymbol{\xi})=1$ for all $\boldsymbol{\xi}$. One easily verifies that this gives all eigenvectors of $U$, with corresponding eigenvalues

$$
\lambda_{S}=\sum_{\boldsymbol{\xi}} U\left(\sum_{\nu=1}^{p} \xi^{\nu}\right) \prod_{\mu \in S} \xi^{\mu}
$$

The eigenvalues depend only on the size $|S|$ of the subset. Application of (25) to our matrices $M$ and $Q$ gives the following eigenvalue spectra:

$$
\lambda_{S}^{M}=c\left\langle\left[\prod_{\mu \in S} \xi^{\mu}\right] \tanh \left[\frac{\beta}{c} \phi\left(\sum_{\nu} \xi_{\nu}\right)\right]\right\rangle_{\boldsymbol{\xi}} \quad \lambda_{S}^{Q}=c\left\langle\left[\prod_{\mu \in S} \xi^{\mu}\right] \tanh ^{2}\left[\frac{\beta}{c} \phi\left(\sum_{\nu} \xi_{\nu}\right)\right]\right\rangle_{\boldsymbol{\xi}}
$$

Clearly, $\lim _{\beta \rightarrow 0} \lambda_{S}^{M}=\lim _{\beta \rightarrow 0} \lambda_{S}^{Q}=0$. For monotonically non-decreasing functions $\phi(x)$, the largest $M$ eigenvalue is found for $|S|=1$, whereas the largest $Q$ eigenvalue corresponds to $S=\emptyset$ :

$$
\begin{aligned}
\lambda_{\text {max }}^{M} & =\frac{c}{p}\left\langle\left(\sum_{\mu} \xi^{\mu}\right) \tanh \left[\frac{\beta}{c} \phi\left(\sum_{\nu} \xi^{\nu}\right)\right]\right\rangle_{\boldsymbol{\xi}} \\
\lambda_{\text {max }}^{Q} & =c\left\langle\tanh ^{2}\left[\frac{\beta}{c} \phi\left(\sum_{\mu} \xi^{\mu}\right)\right]\right\rangle_{\boldsymbol{\xi}}
\end{aligned}
$$




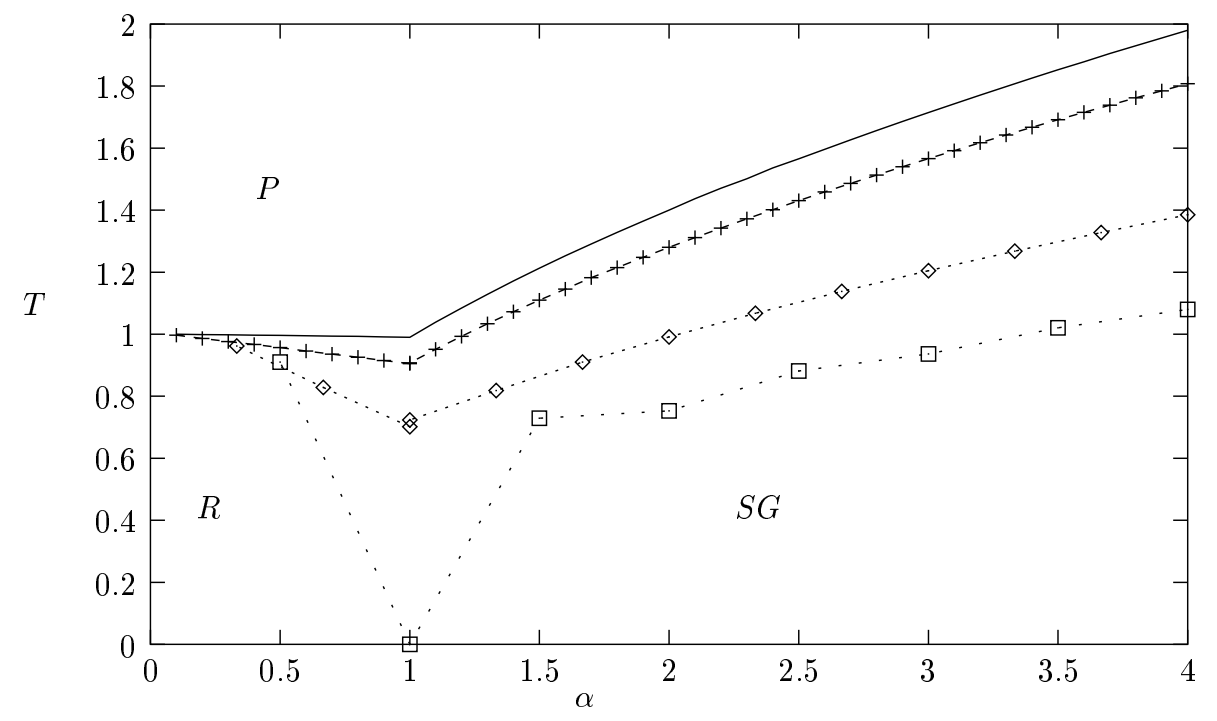

Figure 1. Phase diagram of the finite connectivity Hopfield model, where $\phi(x)=x$. Connected markers give the $\mathrm{P} \rightarrow \mathrm{R}$ and $\mathrm{P} \rightarrow \mathrm{SG}$ transition temperatures in the $(\alpha, T)$ plane (where $\alpha=p / c$ ), for $c=2(\square), c=3(\diamond), c=10(+)$, whereas the $c=100$ transition is indicated by a solid line. The transition line for $c=100$ is nearly indistinguishable from the corresponding $c \rightarrow \infty$ line segments $T=1$ (for $\alpha<1$ ) and $T=\sqrt{\alpha}($ for $\alpha>1)$.

This leads us to the following equations for the $\mathrm{P} \rightarrow \mathrm{R}$ and $\mathrm{P} \rightarrow \mathrm{SG}$ transition lines:

$$
\begin{array}{ll}
\mathrm{P} \rightarrow \mathrm{R}: & \frac{c}{p} 2^{-p} \sum_{n=0}^{p}\left(\begin{array}{l}
p \\
n
\end{array}\right)(p-2 n) \tanh \left[\frac{\beta}{c} \phi(p-2 n)\right]=1 \\
\mathrm{P} \rightarrow \mathrm{SG}: & c 2^{-p} \sum_{n=0}^{p}\left(\begin{array}{l}
p \\
n
\end{array}\right) \tanh ^{2}\left[\frac{\beta}{c} \phi(p-2 n)\right]=1
\end{array}
$$

Equations (2829) will generally have to be solved numerically.

We may finally inspect the limit $c \rightarrow \infty$, with $\alpha=p / c$ fixed, where we expect to find a generalization of the results in [6]. The central limit theorem now allows us to replace $p^{-\frac{1}{2}} \sum_{\mu=1}^{p} \xi^{\mu}$ by a zero-average and unit-variance Gaussian variable, so (26 27) simplify to (provided the limits exist, and with $D y=(2 \pi)^{-\frac{1}{2}} e^{-\frac{1}{2} y^{2}}$ ):

$$
\begin{aligned}
& \lim _{p \rightarrow \infty} \lambda_{\max }^{M}=\frac{1}{\alpha} \int D y y \lim _{p \rightarrow \infty}\left\{\sqrt{p} \tanh \left[\frac{\alpha \beta}{p} \phi(y \sqrt{p})\right]\right\} \\
& \lim _{p \rightarrow \infty} \lambda_{\max }^{Q}=\frac{1}{\alpha} \int D y \lim _{p \rightarrow \infty}\left\{p \tanh ^{2}\left[\frac{\alpha \beta}{p} \phi(y \sqrt{p})\right]\right\}
\end{aligned}
$$

\subsection{Application to Specific Synaptic Kernels}

We now have to make explicit choices for the kernel $\phi(x)$ in our expression for the interaction matrix, viz. $J_{i j}=\frac{c_{i j}}{c} \phi\left(\boldsymbol{\xi}_{i} \cdot \boldsymbol{\xi}_{j}\right)$. We first inspect the finite connectivity Hopfield model $J_{i j}=\frac{c_{i j}}{c} \boldsymbol{\xi}_{i} \cdot \boldsymbol{\xi}_{j}$. Here we have $\phi(x)=x$, and the $c \rightarrow \infty$ expressions 


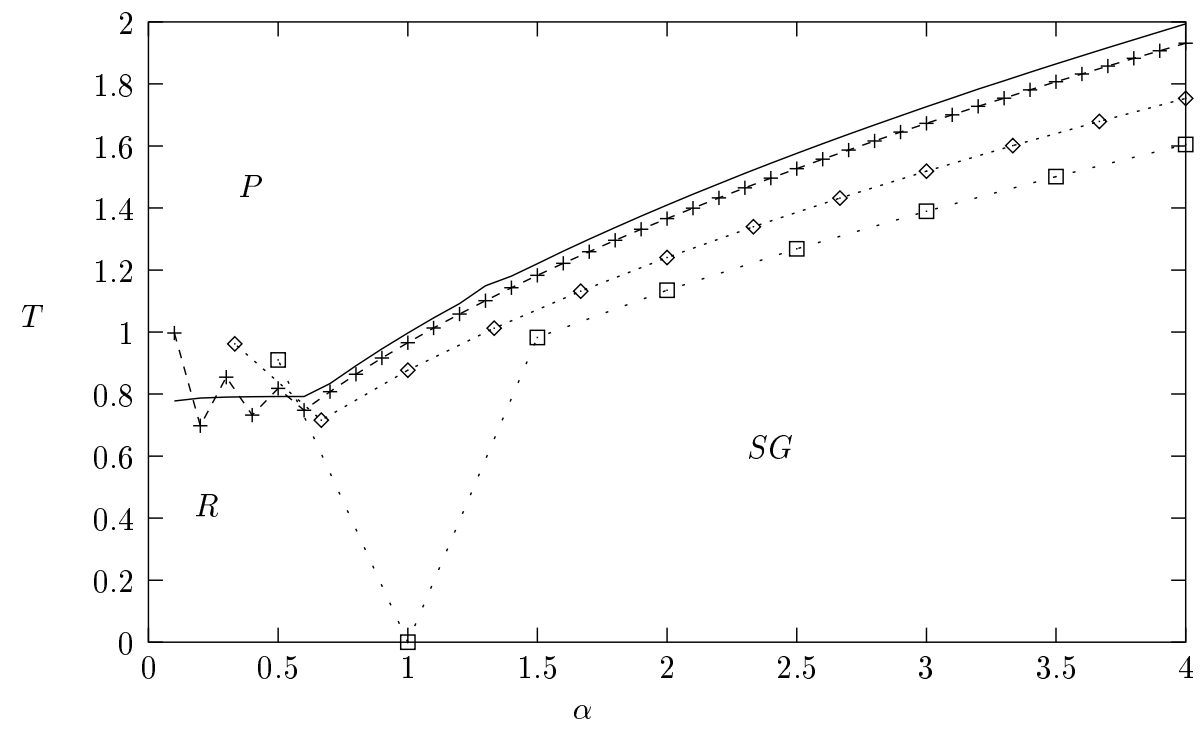

Figure 2. Phase diagram of the finite connectivity clipped Hopfield model, where $\phi(x)=\sqrt{p} \operatorname{sgn}(x)$. Connected markers give the $\mathrm{P} \rightarrow \mathrm{R}$ and $\mathrm{P} \rightarrow \mathrm{SG}$ transition temperatures in the $(\alpha, T)$ plane (where $\alpha=p / c)$, for $c=2(\square), c=3(\diamond), c=10$ $(+)$, whereas the $c=100$ transition is indicated by a solid line. The transition line for $c=100$ is again nearly indistinguishable from the corresponding $c \rightarrow \infty$ line segments, here given by $T=\sqrt{2 / \pi}$ (for $\alpha<\sqrt{2 / \pi}$ ) and $T=\sqrt{\alpha}$ (for $\alpha>\sqrt{2 / \pi}$ ).

for the $\mathrm{P} \rightarrow \mathrm{R}$ and $\mathrm{P} \rightarrow \mathrm{SG}$ transition temperatures (30)31) reduce to $T_{R}=1$ and $T_{S G}=\sqrt{\alpha}$ (in accordance with the extreme dilution results in [6]). In addition one finds that for $c \rightarrow \infty$ the effective field distributions $W_{\boldsymbol{\xi}}(h)$ become Gaussian, with mean $\bar{h}_{\boldsymbol{\xi}}=\left\langle\int d h W_{\boldsymbol{\xi}^{\prime}} \tanh (\beta h)\left(\boldsymbol{\xi} \cdot \boldsymbol{\xi}^{\prime}\right)\right\rangle_{\boldsymbol{\xi}^{\prime}}$ and variance $\sigma^{2}=\alpha\left\langle\int d h W_{\boldsymbol{\xi}^{\prime}} \tanh ^{2}(\beta h)\left(\boldsymbol{\xi} \cdot \boldsymbol{\xi}^{\prime}\right)\right\rangle_{\boldsymbol{\xi}^{\prime}}-\bar{h}_{\boldsymbol{\xi}}^{2}$, from which one immediately recovers the RS order parameter equations of [6] (describing states with a single 'condensed' pattern, for the scaling regime $c \rightarrow \infty$ with $c / N \rightarrow 0)$ :

$$
m=\int D y \tanh [\beta(m+y \sqrt{\alpha q})] \quad q=\int D y \tanh ^{2}[\beta(m+y \sqrt{\alpha q})]
$$

The results of solving (2829) numerically for finite $c$ are shown in figure 1. Note that $\alpha=p / c \in\left\{\frac{1}{c}, \frac{2}{c}, \frac{3}{c}, \ldots\right\}$, so only the actual markers in figure 1 correspond to physically realizable parameter values (the connecting line segments just provide a guide to the eye). We observe that for $c=100$ the transition lines away from the paramagnetic phase are already nearly identical to those corresponding to $c \rightarrow \infty$. For $c=2$, the system remains in a paramagnetic phase up to $T=0$ for $\alpha=1$. Here, interestingly, for $\alpha=0.5$ (i.e. $p=1$ ) there is a transition to a nonzero retrieval overlap at finite $T$. This is not what one would find in e.g. a $1-d$ Ising chain, in which the connectivity is $c=2$, but where there is no phase transition at finite $T$; the difference is due to the fact that in our present model the connectivity equals 2 only on average. Note that the value $c=1$ corresponds to the percolation transition [12. We will turn to the location of the more complicated $\mathrm{R} \rightarrow \mathrm{SG}$ transition in a subsequent section. 


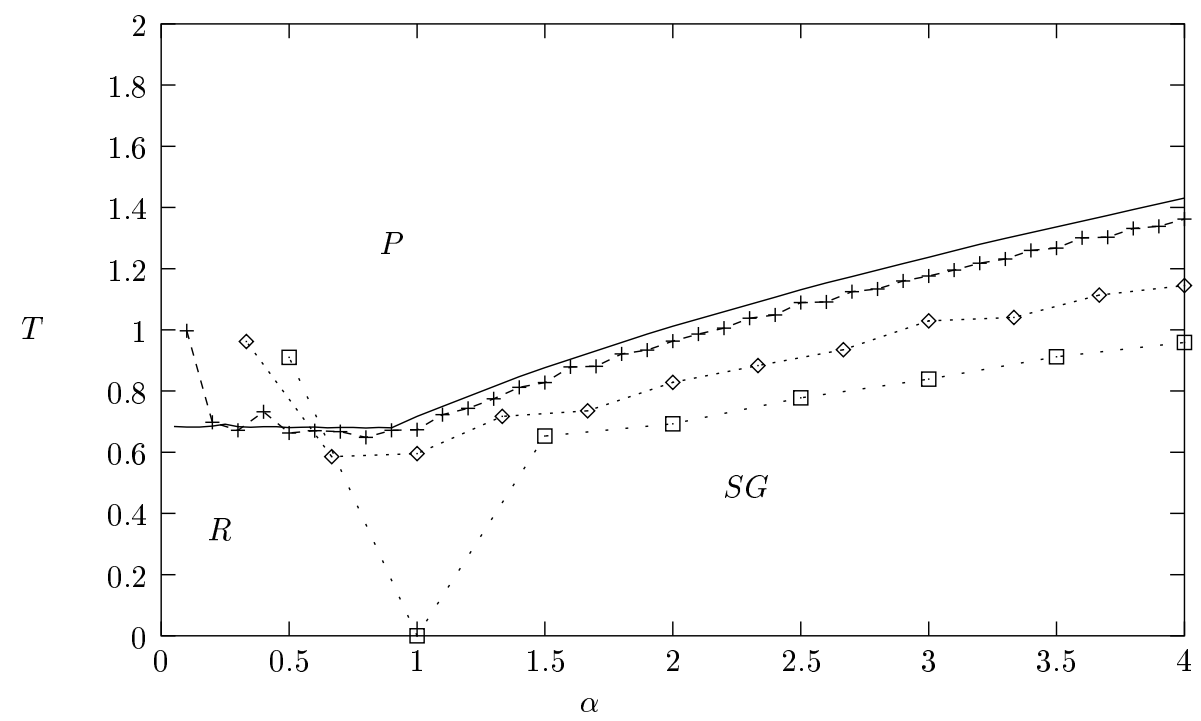

Figure 3. Phase diagram of a model intermediate between the previous two, with $\phi(x)=x$ for $|x|<\sqrt{p}$ and $\phi(x)=\sqrt{p} \operatorname{sgn}(x)$ for $|x|>\sqrt{p}$. Connected markers give the $\mathrm{P} \rightarrow \mathrm{R}$ and $\mathrm{P} \rightarrow \mathrm{SG}$ transition temperatures in the $(\alpha, T)$ plane, for $c=2(\square), c=3(\diamond)$, $c=10(+)$, whereas the $c=100$ transition is indicated by a solid line. The transition line for $c=100$ is again nearly indistinguishable from the corresponding $c \rightarrow \infty$ line segments, here given by $T=\operatorname{Erf}\left(1 / \sqrt{2}\right.$ ) (for small $\alpha$ ) and $T=[\alpha(1-\sqrt{2 / \pi e})]^{\frac{1}{2}}$ (for large $\alpha)$.

Our second application is obtained upon choosing a finite connectivity version of the so-called clipped Hebbian synapses: $J_{i j}=\frac{c_{i j}}{c} \sqrt{p} \operatorname{sgn}\left(\boldsymbol{\xi}_{i} \cdot \boldsymbol{\xi}_{j}\right)$ (the specific scaling with $\sqrt{p}$ ensures a proper limit $c \rightarrow \infty)$. Here $\phi(x)=\sqrt{p} \operatorname{sgn}(x)$, and the $c \rightarrow \infty$ expressions for the $\mathrm{P} \rightarrow \mathrm{R}$ and $\mathrm{P} \rightarrow \mathrm{SG}$ transition temperatures (30.31) now reduce to $T_{R}=\sqrt{2 / \pi}$ and $T_{S G}=\sqrt{\alpha}$. The results of solving (2829) numerically for finite $c$ are shown in figure 2 As before, only the actual markers in figure 2 correspond to physically realizable parameter values. As was the case for full connectivity [28, also with finite connectivity one finds, surprisingly from an information processing point of view, that the differences between Hebbian and clipped Hebbian synapses are only of a quantitative nature; limited largely to a rescaling of critical temperatures.

Our third and final finite connectivity network example is one which interpolates between Hebbian and clipped Hebbian synapses: $\phi(x)=x$ (i.e. Hebbian) for $|x|<\sqrt{p}$, and $\phi(x)=\sqrt{p} \operatorname{sgn}(x)$ (i.e. clipped Hebbian) for $|x| \geq \sqrt{p}$. The value $\sqrt{p}$ is found to be the natural and most interesting scaling for the cut-off point in this definition. The $c \rightarrow \infty$ expressions for the $\mathrm{P} \rightarrow \mathrm{R}$ and $\mathrm{P} \rightarrow \mathrm{SG}$ transition temperatures (30 31) now reduce to $T_{R}=\operatorname{Erf}(1 / \sqrt{2})$ and $T_{S G}=[\alpha(1-\sqrt{2 / \pi e})]^{\frac{1}{2}}$. The results of solving (2829) numerically for finite $c$ are shown in figure 3. Again only the markers correspond to physically realizable parameter values. The apparent irregularities in the transition lines are due to the cut-off in the function $\phi(x)$ at $\left|\boldsymbol{\xi} \cdot \boldsymbol{\xi}^{\prime}\right|=\sqrt{p}$. 


\section{RS Ground State of the Finite Connectivity Hopfield Model}

For large $\alpha$, one expects replica symmetry to break at low temperatures. In contrast to full connectivity models, for finite connectivity models RSB theory is still under development. Here we will therefore analyze as yet only the $T \rightarrow 0$ limit of our RS equations. Apart from giving exact statements at least for small $\alpha$, a calculation of the RS ground state entropy leads for large $\alpha$ to a bound for the location of the RSB transition in the phase diagram, since the latter must come before the zero entropy line.

\subsection{RS Order Parameters at $T=0$}

We follow closely the RS ground state analysis carried out for finite connectivity spin glasses [12, 13]. At $T=0$ there are no thermal fluctuations, so the effective fields in (14) are identical to the true local fields. The latter can take only discrete values, due to the finite number of connections per spin, hence each $W_{\boldsymbol{\xi}}(h)$ is a sum of delta peaks:

$$
W_{\boldsymbol{\xi}}(h)=\sum_{\ell=-\infty}^{\infty} K_{\boldsymbol{\xi}}(\ell) \delta\left(h-\frac{\ell}{c}\right)
$$

Upon taking the limit $\beta \rightarrow \infty$ in (19), using $\lim _{\beta \rightarrow \infty} \beta^{-1} \tanh ^{-1}[\tanh (\beta x) \tanh (\beta y)]=$ $\frac{1}{2}|x+y|-\frac{1}{2}|x-y|$, one verifies by substitution that (32) is indeed a solution. Insertion into the right hand side of (19) gives (with $\bar{\delta}_{n m}=1-\delta_{n m}$ ):

$$
\begin{aligned}
& W_{\boldsymbol{\xi}}(h)=\int \frac{d y}{2 \pi} e^{-i y h} \exp \left(c \left\langle\left\{\sum_{\ell^{\prime}=1}^{\left|\boldsymbol{\xi} \cdot \boldsymbol{\xi}^{\prime}\right|-1}\left[K_{\boldsymbol{\xi}^{\prime}}\left(\ell^{\prime}\right) e^{\frac{i y \ell^{\prime}}{c} \operatorname{sgn}\left(\boldsymbol{\xi} \cdot \boldsymbol{\xi}^{\prime}\right)}+K_{\boldsymbol{\xi}^{\prime}}\left(-\ell^{\prime}\right) e^{-\frac{i y \ell^{\prime}}{c} \operatorname{sgn}\left(\boldsymbol{\xi} \cdot \boldsymbol{\xi}^{\prime}\right)}\right]\right.\right.\right. \\
& \left.\left.\left.+\sum_{\ell^{\prime}=\left|\boldsymbol{\xi} \cdot \boldsymbol{\xi}^{\prime}\right|}^{\infty}\left[K_{\boldsymbol{\xi}^{\prime}}\left(\ell^{\prime}\right) e^{\frac{i y}{c} \boldsymbol{\xi} \boldsymbol{\xi}^{\prime}}+K_{\boldsymbol{\xi}^{\prime}}\left(-\ell^{\prime}\right) e^{-\frac{i y}{c} \boldsymbol{\xi} \boldsymbol{\xi}^{\prime}}\right]-\left[1-K_{\left.\boldsymbol{\xi}^{\prime}(0)\right]}\right\} \bar{\delta}_{\boldsymbol{\xi} \cdot \boldsymbol{\xi}_{, 0}^{\prime}}\right]\right\rangle_{\boldsymbol{\xi}^{\prime}}\right)
\end{aligned}
$$

Upon expanding the exponent in the right-hand side, we would indeed recognize a sum of delta peaks. Integrating of both sides over an infinitesimally small interval around $h=\ell / c$ leads to equations for the factors $K_{\boldsymbol{\xi}}^{ \pm}(\ell)$ :

$$
\begin{aligned}
K_{\boldsymbol{\xi}}(\ell)= & \lim _{\epsilon \rightarrow 0} \int \frac{d z}{\pi z} \sin (z) e^{-\frac{i \ell z}{c \epsilon}} \exp \left(c \left\langle\left\{\sum_{\ell^{\prime}=1}^{\left|\boldsymbol{\xi} \cdot \boldsymbol{\xi}^{\prime}\right|-1}\left[K_{\boldsymbol{\xi}^{\prime}}\left(\ell^{\prime}\right) e^{\frac{i z \ell^{\prime}}{c \epsilon} \operatorname{sgn}\left(\boldsymbol{\xi} \cdot \boldsymbol{\xi}^{\prime}\right)}+K_{\boldsymbol{\xi}^{\prime}}\left(-\ell^{\prime}\right) e^{-\frac{i z \ell^{\prime}}{c \epsilon} \operatorname{sgn}\left(\boldsymbol{\xi} \cdot \boldsymbol{\xi}^{\prime}\right)}\right]\right.\right.\right. \\
& \left.\left.\left.+\sum_{\ell^{\prime}=\left|\boldsymbol{\xi} \cdot \boldsymbol{\xi}^{\prime}\right|}^{\infty}\left[K_{\boldsymbol{\xi}^{\boldsymbol{B}^{\prime}}}\left(\ell^{\prime}\right) e^{\frac{i z}{c \epsilon} \boldsymbol{\xi} \boldsymbol{\xi}^{\prime}}+K_{\boldsymbol{\xi}^{\prime}}\left(-\ell^{\prime}\right) e^{-\frac{i z}{c \epsilon}} \boldsymbol{\xi} \boldsymbol{\xi}^{\prime}\right]-\left[1-K_{\boldsymbol{\xi}^{\prime}}(0)\right]\right\} \bar{\delta}_{\boldsymbol{\xi} \cdot \boldsymbol{\xi}^{\prime}, 0}\right\rangle_{\boldsymbol{\xi}^{\prime}}\right)
\end{aligned}
$$

For $\epsilon \rightarrow 0$, the periodic part of the integral (involving $z / c \epsilon$ ) oscillates rapidly and decouples from the non-oscillating part. We note that (34) is of the form $\lim _{\epsilon \rightarrow 0} \int \frac{d z}{\pi z} \sin (z) f(z / \epsilon)$, where $f(z+2 \pi)=f(z)$. The function $f$ may be written as a Fourier series, $f(z / \epsilon)=\sum_{n=-\infty}^{\infty} a_{n} e^{i n z / \epsilon}$. Consequently:

$$
\lim _{\epsilon \rightarrow 0} \int \frac{d z}{\pi z} \sin (z) f\left(\frac{z}{\epsilon}\right)=a_{0} \int \frac{d z}{\pi z} \sin (z)+\sum_{n \neq 0} a_{n} \lim _{\epsilon \rightarrow 0} \int \frac{d z}{\pi z} \sin (\epsilon z) e^{i n z}=a_{0}
$$


Since $a_{0}=(2 \pi)^{-1} \int_{-\pi}^{\pi} d \phi f(\phi)$, expression (34) can be simplified to

$$
\begin{aligned}
& \left.\left.K_{\boldsymbol{\xi}}(\ell)=e^{-c\langle[1-K} \boldsymbol{\xi}^{\prime}(0)\right] \bar{\delta} \boldsymbol{\xi} \cdot \boldsymbol{\xi}_{, 0}^{\prime}\right\rangle \boldsymbol{\xi}^{\prime} \int_{-\pi}^{\pi} \frac{d \phi}{2 \pi} \exp \left(c \left\langle\left\{\sum_{\ell^{\prime}=1}^{\left|\boldsymbol{\xi} \cdot \boldsymbol{\xi}^{\prime}\right|-1}\left[K_{\boldsymbol{\xi}^{\prime}}\left(\ell^{\prime}\right)+K_{\boldsymbol{\xi}^{\prime}}\left(-\ell^{\prime}\right)\right] \cos \left(\ell^{\prime} \phi\right)\right.\right.\right. \\
& \left.\left.\left.+\sum_{\ell^{\prime}=\left|\boldsymbol{\xi} \cdot \boldsymbol{\xi}^{\prime}\right|}^{\infty}\left[K_{\boldsymbol{\xi}^{\prime}}\left(\ell^{\prime}\right)+K_{\boldsymbol{\xi}^{\prime}}\left(-\ell^{\prime}\right)\right] \cos \left(\boldsymbol{\xi} \cdot \boldsymbol{\xi}^{\prime} \phi\right)\right\} \bar{\delta}_{\boldsymbol{\xi} \cdot \boldsymbol{\xi}_{, 0}^{\prime}}\right\rangle_{\boldsymbol{\xi}^{\prime}}\right) \\
& \times \cos \left(-\ell \phi+c\left\langle\left\{\sum_{\ell^{\prime}=1}^{\left|\boldsymbol{\xi} \cdot \boldsymbol{\xi}^{\prime}\right|-1}\left[K_{\boldsymbol{\xi}^{\prime}}\left(\ell^{\prime}\right)-K_{\boldsymbol{\xi}^{\prime}}\left(-\ell^{\prime}\right)\right] \sin \left(\ell^{\prime} \phi\right) \operatorname{sgn}\left(\boldsymbol{\xi} \cdot \boldsymbol{\xi}^{\prime}\right)\right.\right.\right. \\
& \left.\left.\left.+\sum_{\ell^{\prime}=\left|\boldsymbol{\xi} \cdot \boldsymbol{\xi}^{\prime}\right|}^{\infty}\left[K_{\boldsymbol{\xi}^{\prime}}\left(\ell^{\prime}\right)-K_{\boldsymbol{\xi}^{\prime}}\left(-\ell^{\prime}\right)\right] \sin \left(\boldsymbol{\xi} \cdot \boldsymbol{\xi}^{\prime} \phi\right)\right\} \bar{\delta}_{\boldsymbol{\xi} \cdot \boldsymbol{\xi}_{, 0}^{\prime}}\right\rangle_{\boldsymbol{\xi}^{\prime}}\right)
\end{aligned}
$$

Solutions to this set of equations can be found numerically, via iteration.

\section{2. $R S$ Entropy at $T=0$}

We next calculate the RS zero temperature entropy per spin $s_{0}$, by expanding the free energy per spin up to order $T$ :

$$
f=f_{T=0}-s_{0} T+\mathcal{O}\left(T^{2}\right)
$$

Care is needed in taking the temperature derivative of the free energy, in view of the normalization constraint on the order parameters $W_{\boldsymbol{\xi}}(h)$. One generally has

$$
\frac{d f}{d T}=\left.\frac{\partial f[\ldots]}{\partial T}\right|_{\text {saddle }}+\left.\sum_{\boldsymbol{\xi}^{\prime}} \int d h^{\prime} \frac{\delta f[\ldots]}{\delta W_{\boldsymbol{\xi}^{\prime}}\left(h^{\prime}\right)}\right|_{\text {saddle }} \frac{\partial W_{\boldsymbol{\xi}^{\prime}}^{\star}\left(h^{\prime}\right)}{\partial T}
$$

where $W^{\star}$ denotes the saddle-point of $f[\ldots]$. In contrast to unconstrained extremization, the functional derivative in the right hand side of (37) need not vanish, since extremization of $f$ is restricted to the subspace in which $\int d h W_{\boldsymbol{\xi}}(h)=1$ :

$$
\frac{\delta f[\ldots]}{\delta W_{\boldsymbol{\xi}}(h)}+\sum_{\boldsymbol{\xi}^{\prime}} \lambda_{\boldsymbol{\xi}^{\prime}} \frac{\delta}{\delta W_{\boldsymbol{\xi}}(h)}\left[\int d h^{\prime} W_{\boldsymbol{\xi}^{\prime}}\left(h^{\prime}\right)-1\right]=0
$$

Here $\left\{\lambda_{\boldsymbol{\xi}^{\prime}}\right\}$ are Lagrange multipliers. As a consequence, we find $\delta f[\ldots] / \delta W_{\boldsymbol{\xi}}(h)=\lambda_{\boldsymbol{\xi}}$ at the saddle point (i.e. independent of the field $h$ ), and hence

$$
\frac{d f}{d T}=\left.\frac{\partial f[\ldots]}{\partial T}\right|_{\text {saddle }}+\sum_{\boldsymbol{\xi}^{\prime}} \lambda_{\boldsymbol{\xi}^{\prime}} \frac{\partial}{\partial T} \int d h^{\prime} W_{\boldsymbol{\xi}^{\prime}}^{\star}\left(h^{\prime}\right)=\left.\frac{\partial f[\ldots]}{\partial T}\right|_{\text {saddle }}
$$

For the purpose of calculating $s_{0}$, we may thus simply insert the $T=0$ saddle-point (3235) into our expression (20) for the free energy, giving:

$$
s_{0}=\lim _{\beta \rightarrow \infty} \beta^{2} \frac{\partial}{\partial \beta}\left\langle\left\langle\frac{c}{2 \beta} \sum_{\ell, \ell^{\prime} \neq 0} K_{\boldsymbol{\xi}}(\ell) K_{\boldsymbol{\xi}^{\prime}}\left(\ell^{\prime}\right) \log \left[1+\tanh \left(\frac{\beta \ell}{c}\right) \tanh \left(\frac{\beta \ell^{\prime}}{c}\right) \tanh \left(\frac{\beta}{c} \boldsymbol{\xi} \cdot \boldsymbol{\xi}^{\prime}\right)\right]\right.\right.
$$


Finite Connectivity Attractor Neural Networks

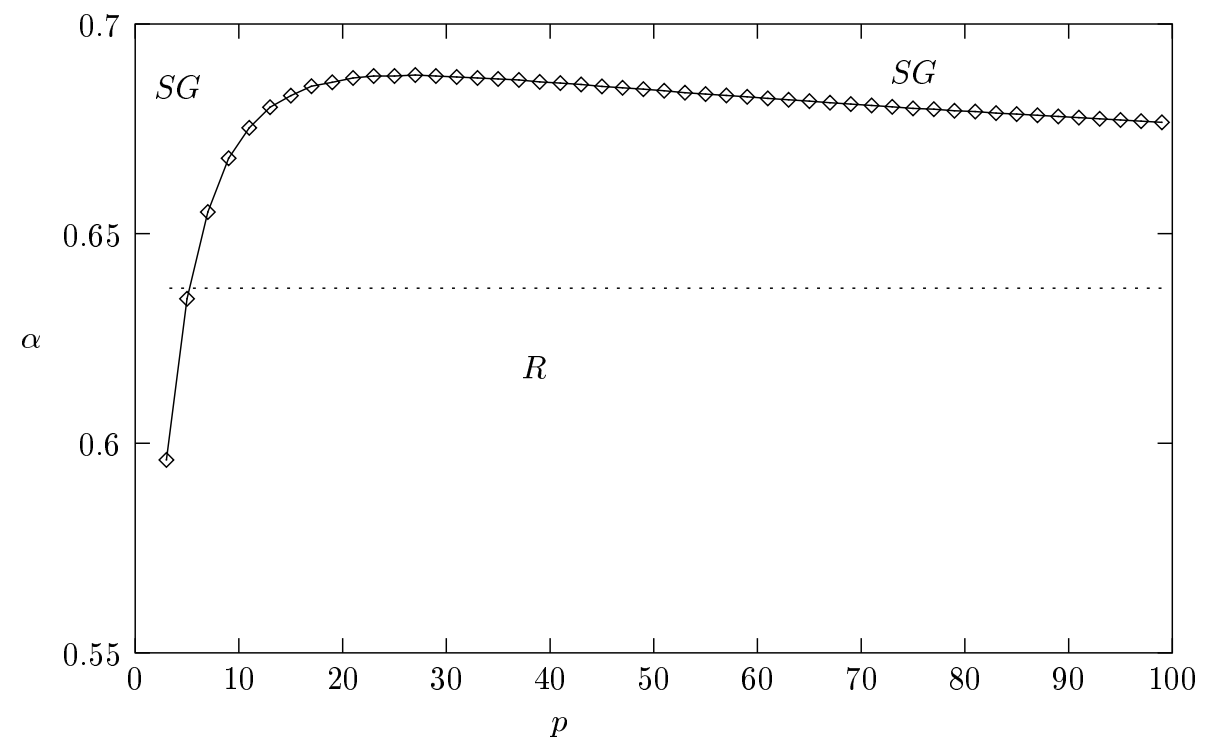

Figure 4. Zero temperature phase diagram of the finite connectivity Hopfield model, in RS approximation and for odd $p$ (note: $p=\alpha c$ ). Connected diamonds $(\diamond)$ : the $\mathrm{R} \rightarrow \mathrm{SG}$ transition line. Dashed horizontal line: the $c \rightarrow \infty$ location for the $\mathrm{R} \rightarrow \mathrm{SG}$ line, $\alpha_{c}=2 / \pi$, as known from [6].

$$
\begin{gathered}
-\frac{c}{2 \beta} \sum_{\ell \neq 0} K_{\boldsymbol{\xi}}(\ell)\left[\log \left[1+\tanh \left(\frac{\beta \ell}{c}\right) \tanh \left(\frac{\beta}{c} \boldsymbol{\xi} \cdot \boldsymbol{\xi}^{\prime}\right)\right]+\log \left[1-\tanh \left(\frac{\beta \ell}{c}\right) \tanh \left(\frac{\beta}{c} \boldsymbol{\xi} \cdot \boldsymbol{\xi}^{\prime}\right)\right]\right] \\
\left.\left.-\frac{c}{2 \beta} \log \cosh \left(\frac{\beta}{c} \boldsymbol{\xi} \cdot \boldsymbol{\xi}^{\prime}\right)-\frac{1}{\beta} \sum_{\ell \neq 0} K_{\boldsymbol{\xi}}(\ell) \log \left[2 \cosh \left(\frac{\beta \ell}{c}\right)\right]\right\rangle\right\rangle_{\boldsymbol{\xi} \boldsymbol{\xi}^{\prime}} \\
=\frac{c}{2} \log 2\left\langle\sum_{\ell=0}^{\left|\boldsymbol{\xi} \boldsymbol{\xi}^{\prime}\right|-1}\left\{K_{\boldsymbol{\xi}}\left(-\left|\boldsymbol{\xi} \cdot \boldsymbol{\xi}^{\prime}\right|\right)+K_{\boldsymbol{\xi}}\left(\left|\boldsymbol{\xi} \cdot \boldsymbol{\xi}^{\prime}\right|\right)-\left[K_{\boldsymbol{\xi}}(\ell)+K_{\boldsymbol{\xi}}(-\ell)\right]\left[1-\frac{1}{2} \delta_{\ell, 0}\right]\right\}\right. \\
\times\left[K_{\boldsymbol{\xi}^{\prime}}(\ell)+K_{\boldsymbol{\xi}^{\prime}}(-\ell)\right]\left[1-\frac{1}{2} \delta_{\ell, 0}\right] \bar{\delta}_{\left.\left.\boldsymbol{\xi}_{\boldsymbol{\xi}^{\prime}, 0}\right\rangle\right\rangle_{\boldsymbol{\xi} \boldsymbol{\xi}^{\prime}}+\log 2\left\langle K_{\boldsymbol{\xi}}(0)\right\rangle_{\boldsymbol{\xi}}} \\
+\frac{c}{4}[2 \log 2-\log 3]\left\langle\left\langle\left[K_{\boldsymbol{\xi}}\left(-\left|\boldsymbol{\xi} \cdot \boldsymbol{\xi}^{\prime}\right|\right)+K_{\boldsymbol{\xi}}\left(\left|\boldsymbol{\xi} \cdot \boldsymbol{\xi}^{\prime}\right|\right)\right]\right.\right. \\
\left.\left.\times\left[K_{\boldsymbol{\xi}^{\prime}}\left(-\left|\boldsymbol{\xi} \cdot \boldsymbol{\xi}^{\prime}\right|\right)+K_{\boldsymbol{\xi}^{\prime}}\left(\left|\boldsymbol{\xi} \cdot \boldsymbol{\xi}^{\prime}\right|\right)\right] \bar{\delta}_{\boldsymbol{\xi} \cdot \boldsymbol{\xi}^{\prime}, 0}\right\rangle\right\rangle_{\boldsymbol{\xi} \boldsymbol{\xi}^{\prime}}(40)
\end{gathered}
$$

This result is reminiscent of the corresponding expression found for spin glasses in [29].

\subsection{Zero Temperature Phase Transitions}

We next try to solve the coupled equations (35), for randomly drawn patterns, at zero temperature. We know that in the case of perfect retrieval of a pattern $\mu$, half of the sublattice magnetizations (the ones with $\xi^{\mu}=1$ ) must be 1 and the other half must be -1 . At $T=0$ all spins align with their local fields, so it follows from (32) that $K_{\boldsymbol{\xi}}(\ell)=K\left(\xi^{\mu} \ell\right)$ for some set of non-negative numbers $K(\ell)$. We will now choose the

property $K_{\boldsymbol{\xi}}(\ell)=K\left(\xi^{\mu} \ell\right)$ for all $\boldsymbol{\xi} \in\{-1,1\}$ and all integer $\ell$ as an ansatz. Upon 
restricting ourselves for simplicity to odd values of $p$, our equations (35) subsequently simplify to

$$
K(\ell)=e^{-c[1-K(0)]} \int_{-\pi}^{\pi} \frac{d \phi}{2 \pi} e^{c A(\phi,\{K\})} \cos [-\ell \phi+c B(\phi,\{K\})]
$$

with the abbreviations

$$
\begin{aligned}
A(\phi,\{K\})= & 2^{-(p-1)} \sum_{m=(p+1) / 2}^{p}\left(\begin{array}{c}
p \\
m
\end{array}\right) \sum_{\ell=1}^{2 m-p-1}[K(\ell)+K(-\ell)] \cos (\ell \phi) \\
& +2^{-p} \sum_{m=0}^{p}\left(\begin{array}{c}
p \\
m
\end{array}\right) \cos [(p-2 m) \phi] \sum_{\ell=|p-2 m|}^{\infty}[K(\ell)+K(-\ell)] \\
B(\phi,\{K\})= & 2^{-p} \sum_{m=0}^{p} \frac{p-2 m}{p}\left(\begin{array}{c}
p \\
m
\end{array}\right) \sin [(p-2 m) \phi] \sum_{\ell=|p-2 m|}^{\infty}[K(\ell)-K(-\ell)]
\end{aligned}
$$

The result of solving (41) numerically, and subsequently detecting the $\mathrm{R} \rightarrow \mathrm{SG}$ transition (which is found to be first order) is shown in figure 4 in the $(p, \alpha)$ plane. The $\mathrm{R} \rightarrow \mathrm{SG}$ transition line should for $c \rightarrow \infty$ (i.e. $p \rightarrow \infty$ with fixed $\alpha$ ) approach the value $\alpha_{c}=2 / \pi$ $(\approx 0.637)$, in accordance with [6]; the latter limit is shown in the figure as a horizontal dashed line, which is indeed seen to be approached by our $\mathrm{R} \rightarrow \mathrm{SG}$ line as $p \rightarrow \infty$. As usual for the present type of system, we concentrated on bifurcation points. Due to the extensive energy barriers between the ergodic sectors in attractor neural networks, on the important time-scales local stability is more important than thermodynamic stability, so the thermodynamic transition lines (based on comparing values of free energies) are not relevant. Numerical evaluation of the free energy of the candidate solution in fact reveals that the bifurcation lines coincide with the thermodynamic transitions.

Figure 4 shows re-entrance phenomena. It should be noted that an increase of $p$ for fixed $\alpha$ implies a simultaneous increase of the number of stored patterns (with a detrimental effect on recall quality) and of the connectivity $c$ (expected to have a positive effect on recall quality); the non-monotonic appearance of the $\mathrm{R} \rightarrow \mathrm{SG}$ line reflects the competition of these opposite tendencies.

Finally, for the present type of zero temperature solution, our expression for the RS zero temperature entropy (40) reduces to

$$
\begin{aligned}
\frac{s_{0}}{\log 2}= & K(0)+c 2^{-p} \sum_{m=(p+1) / 2}^{p}\left(\begin{array}{c}
p \\
m
\end{array}\right)\left\{\sum_{\ell=0}^{2 m-p-1}[K(2 m-p)+K(p-2 m)\right. \\
- & {\left.\left.[K(\ell)+K(-\ell)]\left[1-\frac{1}{2} \delta_{\ell, 0}\right]\right][K(\ell)+K(-\ell)]\left[1-\frac{1}{2} \delta_{\ell, 0}\right]\right\} } \\
& +c 2^{-p}\left(1-\frac{\log 3}{2 \log 2}\right) \sum_{m=(p+1) / 2}^{p}\left(\begin{array}{c}
p \\
m
\end{array}\right)[K(2 m-p)+K(p-2 m)]^{2}
\end{aligned}
$$

Numerical evaluation of this expression reveals that the RS entropy at $T=0$ is never zero, for any combination of $\alpha$ and $p$. This at first sight somewhat surprising result is in fact in accordance with a similar observation made recently for finite connectivity spin-glasses in [29]. It does, however, not imply that replica symmetry is not broken at low temperatures (which we know must happen for $c \rightarrow \infty[6]$ ). 


\section{Discussion}

In this paper we have shown how the mathematical framework of finite connectivity spin glass replica theory (involving order parameter functions in replica space, rather than matrices) can be combined with the concept of sub-lattices in order to solve a large class of finite connectivity Hopfield-type attractor neural network models near saturation. So far such networks appear to have been studied only in the two technically simpler regimes of full connectivity and so-called extreme dilution, both of which involve a diverging number of bonds per spin $c$ in the thermodynamic limit. We have restricted ourselves to a replica symmetric (RS) analysis. The replica symmetric theory is found to lead to $2^{p}$ coupled integral equations (19), whose solutions give the effective local field distributions in each of the $2^{p}$ sublattices of the system. Here $p$ denotes the number of stored patterns. In the limit $c \rightarrow \infty$ our equations are shown to reduce to the theory of so-called extremely diluted attractor neural networks, as expected. For $T \rightarrow 0$ and below a certain critical value for $\alpha$ one should expect replica symmetry no longer to hold (it has been shown for $c \rightarrow \infty$ in [6]); going beyond replica symmetry would require extending the theory to include one or more steps of replica symmetry breaking, similar to the finite connectivity spin glass calculations in [16, 24, 25, 26, 17, 27].

As is usual for attractor neural networks near saturation, our phase diagrams exhibit three phases: a paramagnetic phase $(\mathrm{P})$, a retrieval phase $(\mathrm{R})$, and a spin-glass phase (SG). We have calculated, for arbitrary values of the number of bonds $c$ per spin and a large class of Hebbian type synaptic kernels, the (second order) $\mathrm{P} \rightarrow \mathrm{R}$ and $\mathrm{P} \rightarrow \mathrm{SG}$ transition lines in the $(\alpha, T)$ phase diagram. For the main member of our model class, the finite connectivity Hopfield model, we have also calculated the (second order) $\mathrm{R} \rightarrow \mathrm{SG}$ transition line at $T=0$. We find that the values of the average connectivity $c$ needed for the system to function as an attractor neural network are surprisingly small, barely exceeding the percolation threshold, even for clipped Hebbian synapses (where each bond carries only one bit of information). Figures 1 to 3 , for instance, underline that already for $c=3$ the phase diagrams differ only in a relatively modest sense from those corresponding to $c \rightarrow \infty$. This underlines the robustness of recurrent neural networks as information processing systems.

Apart from the obvious extension of our present work, the inclusion of RSB solutions of our saddle-point equations and calculation of AT lines [30], it would also be an interesting challenge to attempt a dynamical solution. Within the generating functional analysis formalism of 31] this would for finite connectivity systems involve an effective single spin problem, with order parameters describing single-spin path probabilities and the effect on these path probabilities of single-spin external field perturbations.

\section{Acknowledgement}

One of the authors (BW) gratefully acknowledges helpful discussions with Prof JRL De Almeida. 


\section{References}

[1] Hopfield J J 1982 Proc. Natl. Acad. Sci. USA 792554

[2] Mezard M, Parisi G and Virasoro M A 1987 Spin Glass Theory and Beyond (Singapore: World Scientific)

[3] Amit D J, Gutfreund H and Sompolinsky H 1985 Phys. Rev. A 321007

[4] Amit D J, Gutfreund H and Sompolinsky H 1985 Phys. Rev. Lett. 551530

[5] Derrida B, Gardner E and Zippelius A 1987 Europhys. Lett. 4167

[6] Watkin T L H and Sherrington D 1991 Europhys. Lett. 14791

[7] Skantzos N S and Coolen A C C 2000 J. Phys. A 331841

[8] Skantzos N S and Coolen A C C 2001 J. Phys. A 34929

[9] Coolen A C C 2001 in Handbook of Biological Physics Vol 4 (Elsevier Science; eds. F. Moss and S. Gielen) 531

[10] Coolen A C C 2001 in Handbook of Biological Physics Vol 4 (Elsevier Science; eds. F. Moss and S. Gielen) 597

[11] Viana L and Bray A J 1985 J. Phys. C 183037

[12] Kanter I and Sompolinsky H 1987 Phys. Rev. Lett. 58164

[13] Mezard M and Parisi G 1987 Europhys. Lett. 31067

[14] Mottishaw P and De Dominicis C 1987 J. Phys. A 20 L375

[15] van Hemmen J L and Kühn R 1986 Phys. Rev. Lett. 57913

[16] Wong K Y and Sherrington D 1988 J. Phys. A 21 L459

[17] Monasson R 1998 J. Phys. A 31513

[18] Murayama T, Kabashima Y, Saad D and Vicente R 2000 Phys. Rev. E 621577

[19] Nishimori H 2001 Statistical Physics of Spin Glasses and Information Processing (Oxford: University Press)

[20] Kirkpatrick S and Selman B 1994 Science 2641297

[21] Monasson R and Zecchina R 1998 Phys. Rev. E 561357

[22] Monasson R and Zecchina R 1998 J. Phys. A 319209

[23] Monasson R, Zecchina R, Kirkpatrick S, Selman B and Troyansky L 1999 Nature 400133

[24] de Dominicis C and Goldschmidt Y Y 1989 J. Phys. A 22 L775

[25] Lai P Y and Goldschmidt Y Y 1990 J. Phys. A 233329

[26] Goldschmidt Y Y and de Dominicis C 1990 Phys. Rev. B 412184

[27] Parisi G and Tria F 2002 preprint cond-mat/0207144

[28] van Hemmen J L 1987 Phys. Rev. A 361959

[29] de Almeida J R L 2002 preprint cond-mat/0208534

[30] De Almeida JRL and Thouless DJ 1978 J. Phys. A 11983

[31] De Dominicis C 1978 Phys. Rev. B 184913 
Finite Connectivity Attractor Neural Networks

\section{Appendix A. Calculation of the RS Free Energy}

In this section we calculate the RS free energy per spin, upon inserting (14) into (13). In doing so we will use the short-hands:

$$
\gamma_{m}=\int_{-\pi / 2}^{\pi / 2} \frac{d \phi}{\pi} \log [\cos (\phi)] \cdot \cos (2 m \phi) \quad m=0, \pm 1, \pm 2, \ldots
$$

They obey:

$$
\sum_{m=-\infty}^{\infty} \gamma_{m} e^{2 a m}=\log \cosh (a)
$$

Taking the limit $n \rightarrow 0$ is again found to impose the need to restrict ourselves to even values of $n$. One then obtains, after some algebra, for the entropic term in (13):

$$
\begin{aligned}
& \lim _{n \rightarrow 0} \frac{1}{\beta n}\left\langle\sum_{\boldsymbol{\sigma}} P_{\boldsymbol{\xi}}(\boldsymbol{\sigma}) \log P_{\boldsymbol{\xi}}(\boldsymbol{\sigma})\right\rangle_{\boldsymbol{\xi}}=\lim _{n \rightarrow 0} \frac{1}{\beta n} \sum_{m=-\infty}^{\infty} \int_{0}^{2 \pi} \frac{d \hat{m}}{2 \pi} e^{i \hat{m} m} \int d h e^{\beta h m}\left[\frac{\cos (\hat{m})}{\cosh (\beta h)}\right]^{n} \\
& \times\left\langle W_{\boldsymbol{\xi}}(h) \log \left[\int d h^{\prime} W_{\boldsymbol{\xi}}\left(h^{\prime}\right) \frac{e^{\beta h^{\prime} m}}{\left[2 \cosh \left(\beta h^{\prime}\right)\right]^{n}}\right]\right\rangle_{\boldsymbol{\xi}} \\
& =\frac{1}{\beta} \sum_{m=-\infty}^{\infty} \gamma_{m} \int d h\left\langle W_{\boldsymbol{\xi}}(h) e^{2 \beta h m} \log \left[\int d h^{\prime} W_{\boldsymbol{\xi}}\left(h^{\prime}\right) e^{2 \beta h^{\prime} m}\right]\right\rangle_{\boldsymbol{\xi}} \\
& -\frac{1}{\beta} \int d h\left\langle W_{\boldsymbol{\xi}}(h) \log [2 \cosh (\beta h)]\right\rangle_{\boldsymbol{\xi}}
\end{aligned}
$$

Similarly, for the energetic term $U$ in (13) one finds:

$$
\begin{gathered}
-\lim _{n \rightarrow 0} \frac{c}{2 \beta n}\left\langle\left\langle\sum_{\boldsymbol{\sigma} \boldsymbol{\sigma}^{\prime}} P_{\boldsymbol{\xi}}(\boldsymbol{\sigma}) P_{\boldsymbol{\xi}^{\prime}}\left(\boldsymbol{\sigma}^{\prime}\right)\left[e^{\frac{\beta}{c} \phi\left(\boldsymbol{\xi} \cdot \boldsymbol{\xi}^{\prime}\right)\left(\boldsymbol{\sigma} \cdot \boldsymbol{\sigma}^{\prime}\right)}-1\right]\right\rangle\right\rangle_{\boldsymbol{\xi} \boldsymbol{\xi}^{\prime}} \\
=-\frac{c}{2 \beta} \int d h d h^{\prime}\left\langle\left\langleW _ { \boldsymbol { \xi } } ( h ) W _ { \boldsymbol { \xi } ^ { \prime } } ( h ^ { \prime } ) \operatorname { l o g } \left[\sum_{\sigma \sigma^{\prime}} e^{\left.\left.\left.\frac{\beta}{c} \phi\left(\boldsymbol{\xi} \cdot \boldsymbol{\xi}^{\prime}\right) \sigma \sigma^{\prime}+\beta\left[h \sigma+h^{\prime} \sigma^{\prime}\right]\right]\right\rangle\right\rangle_{\boldsymbol{\xi} \boldsymbol{\xi}^{\prime}}}\right.\right.\right. \\
+\frac{c}{\beta} \int d h\left\langle W_{\boldsymbol{\xi}}(h) \log \cosh (\beta h)\right\rangle_{\boldsymbol{\xi}} \\
=-\frac{c}{2 \beta} \int d h d h^{\prime}\left\langle\left\langle W_{\boldsymbol{\xi}}(h) W_{\boldsymbol{\xi}^{\prime}}\left(h^{\prime}\right) \log \left[1+\tanh (\beta h) \tanh \left(\beta h^{\prime}\right) \tanh \left(\frac{\beta}{c} \phi\left(\boldsymbol{\xi} \cdot \boldsymbol{\xi}^{\prime}\right)\right)\right]\right\rangle\right\rangle_{\boldsymbol{\xi} \boldsymbol{\xi}^{\prime}} \\
-\frac{c}{2 \beta}\left\langle\left\langle\log \cosh \left[\frac{\beta}{c} \phi\left(\boldsymbol{\xi} \cdot \boldsymbol{\xi}^{\prime}\right)\right]\right\rangle\right\rangle_{\boldsymbol{\xi} \boldsymbol{\xi}^{\prime}}
\end{gathered}
$$

We use (18) to simplify the entropic term (A.2), and add the latter to the energetic term (A.3) to obtain the following expression for the RS free energy per spin:

$$
\begin{aligned}
f_{\mathrm{RS}} & =-\frac{1}{\beta} \int d h\left\langle W_{\boldsymbol{\xi}}(h) \log [2 \cosh (\beta h)]\right\rangle_{\boldsymbol{\xi}}-\frac{c}{2 \beta}\left\langle\left\langle\log \cosh \left[\frac{\beta}{c} \phi\left(\boldsymbol{\xi} \cdot \boldsymbol{\xi}^{\prime}\right)\right]\right\rangle\right\rangle_{\boldsymbol{\xi} \boldsymbol{\xi}^{\prime}} \\
& -\frac{c}{2 \beta} \int d h d h^{\prime}\left\langle\left\langle W_{\boldsymbol{\xi}}(h) W_{\boldsymbol{\xi}^{\prime}}\left(h^{\prime}\right) \log \left[1+\tanh (\beta h) \tanh \left(\beta h^{\prime}\right) \tanh \left(\frac{\beta}{c} \phi\left(\boldsymbol{\xi} \cdot \boldsymbol{\xi}^{\prime}\right)\right)\right]\right\rangle\right\rangle_{\boldsymbol{\xi} \boldsymbol{\xi}^{\prime}} \\
+ & \frac{c}{\beta} \sum_{m=-\infty}^{\infty} \gamma_{m} \int d h d h^{\prime}\left\langle\left\langle W_{\boldsymbol{\xi}}(h) W_{\boldsymbol{\xi}^{\prime}}\left(h^{\prime}\right) e^{2 \beta h m}\left[e^{2 m \tanh ^{-1}\left[\tanh \left(\beta h^{\prime}\right) \tanh \left(\frac{\beta}{c} \phi\left(\boldsymbol{\xi} \cdot \boldsymbol{\xi}^{\prime}\right)\right)\right]}-1\right]\right\rangle\right\rangle_{\boldsymbol{\xi} \boldsymbol{\xi}^{\prime}}
\end{aligned}
$$

At this stage we may use relation A.1 to carry out the summation over $m$. After some further re-arrangement of terms, and application of the simple identity $\cosh \left[\tanh ^{-1}(y)\right]=\left(1-y^{2}\right)^{-1 / 2}$, one then arrives at the final result (20). 